\title{
THREE-DIMENSIONAL COMPUTER MODELLING AND STRESS-STRAIN ANALYSIS OF TEMPOROMANDIBULAR JOINT
}

\author{
Roman Palyvoda \\ Department of Oral and Maxillofacial Surgery \\ Bogomolets National Medical University \\ 1 Zoologichna str., Kyiv, Ukraine, 03680 \\ Romeo.250390@gmail.com \\ Vladyslav Malanchuk \\ Department of Oral and Maxillofacial Surgery \\ Bogomolets National Medical University \\ 1 Zoologichna str., Kyiv, Ukraine, 03680 \\ malanchuk_v_a@ukr.net \\ Mykola Kryschuk \\ Department of Machine Dynamics and Strength of Materials, \\ National Technical University of Ukraine "KPI" \\ 37 Peremogy ave., Kyiv, Ukraine, 03056 \\ Krys@ukr.net \\ Oksana Volovar \\ Department of Oral and Maxillofacial Surgery \\ Bogomolets National Medical University \\ 1 Zoologichna str., Kyiv, Ukraine, 03680 \\ osvolovar@ukr.net
}

\begin{abstract}
The main purpose of the study is to build 3D model of temporomandibular joint and mandible using programs for modelling and design, research stress and strain in cartilage and bone of model depending on different contraction of chewing muscles (especially lateral pterygoid muscle). There were used such methods as computer tomography data, finite element method and analysis topography of lateral pterygoid muscle on cadavers. In results of study was obtained maximal stress in anterior and distal parts of articular disc between 1,4-3 MPa and 0,4-1 MPa. Thus, static loads of articular disc were different, depends on force of contraction lateral pterygoid muscle. Muscle disturbances (hyperfunctioning, atrophy etc.) should be considered in treatment of temporomandibular dysfunction and examined on magnetic resonance investigation if it is present in the same patient.
\end{abstract}

Keywords: temporomandibular joint, lateral pterygoid muscle, finite element analysis, 3D model.

DOI: 10.21303/2504-5679.2016.00088 C Roman Palyvoda, Vladyslav Malanchuk, Mykola Kryschuk, Oksana Volovar

\section{Introduction}

Medicine is an industry that is constantly evolving. At the beginning of $21^{\text {st }}$ century, an integrated approach provides a combination of many related disciplines, which allows taking treatment and getting a positive result in each clinical case.

Computer modeling is a revolutionary introduction to the subject using mathematical and experimental models [1-3]. Finite element method (FEM) was firstly used in dentistry, is an innovative and progressive solution of static and dynamic problems in biological systems including bone structures and cartilage [4-6].

Today, the creation of individual finite-element models is a perspective area of biomechanical research. This method starts to be widely implemented in practice as an important component of planning maxillofacial operations (reconstructive and orthognathic surgery), accurate prediction 
of complex biomechanical reactions of implants, conducting osteotomy and osteosynthesis facial bones etc. $[7,8]$.

The use of finite-element analysis in cases of temporomandibular joint (TMJ) dysfunction and condylar fractures is important, requires high skills in engineering and computer mechanics, knowledge, medical analytics, as expensive equipment and additional methods of examination [9, 10]. Thus, in addition to it also can be used the data obtained from cadavers and MRI measurements [11, 12].

\section{Aim of research}

To build computer three-dimensional model of mandible and TMJ, investigate features of forces and stress-strain distribution making by chewing muscles and improve diagnosis of TMJ's dysfunction using this model.

\section{Materials and methods}

We performed TMJs section from both sides of 3 cadavers used for teaching students at the Department of human anatomy of Bogomolets National medical university. The cadaver's ages ranged from $50-70$ years $(60 \pm 6,5$ years). Cadavers were partially dentate. There were two cadaver's bodies and one cadaver's head.

We used data of computer tomography (Toshiba/Activion 16) and took into account patient with no clinical manifestations of the pathology of maxillofacial area and dysfunction of masticatory system. We applied FEM to analyze distribution of stress and strain with license CAD - CAM programs (Mimics 12.3 (Materialize, Belgium), SolidWorks 2013 x64 Edition (Dassault Systèmes SolidWorks Corp., USA) and Ansys Workbench 14.5 (Ansys Corp., USA) (Fig. 1).
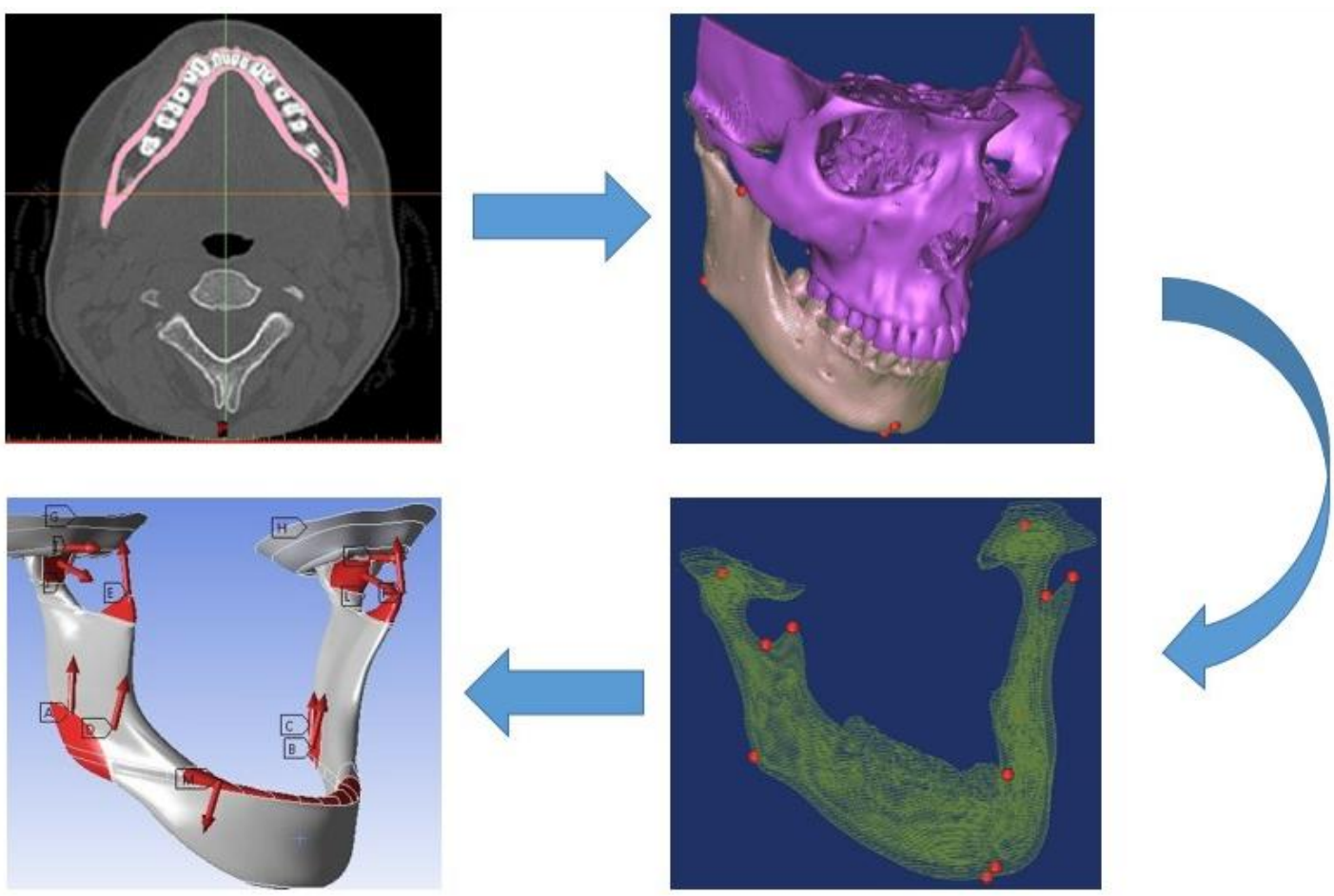

Fig. 1. An algorithm for constructing the finite-element model of the mandible and TMJ

In this way lower jaw and part of temporal bone were cut off other bone of skull, made a correction of circuits (divide or remove individual pixels (voxels)) delete some errors or artefacts. Thereby data of CT was converted into 3D virtual model using program of engineering design and analysis (CAD/CAE - system). We performed usual virtual finite element models with the real parameters. These models made solving goals of our study. 
In semi-automatically mode, we formed border lines of cortical and sponge layers of bone, compared relative measures of articular fossa, condylar process and articular disc of TMJ. Due to Mimics Materialize, 3D model was imported into Solid Works computer software.

Each one from 544 closed lines is composed one to another, have put together sketches, created space and got solid-state model. Used abstraction and simplification of the model geometry, however, created the basic elements of the TMJ [13-16].

Bone and cartilage tissues considered homogeneous, physical and mechanical properties were set in the form of an isotropic material with elastic characteristics and the linear case [17, 18].

For studying the normal biomechanics of the joint, we reviewed including functional loads and contact interactions at the point of contact anatomical structures (Table 1, 2).

Chewing loads simulated by the vector in punctum mobile the muscles of the lower jaw and a single vector of force in the area of the dentition.

Table 1

Standard loads of computer model of chewing muscles (Cartesian coordinate system)

\begin{tabular}{|c|c|c|c|c|}
\hline \multirow{2}{*}{ Chewing muscles } & \multirow{2}{*}{$\mathbf{F}, \mathbf{H}$} & \multicolumn{3}{|c|}{$\begin{array}{c}\text { Components of } \\
\text { Cartesian coordinate system }\end{array}$} \\
\hline & & $\mathbf{X}$ & $\mathbf{Y}$ & $\mathbf{Z}$ \\
\hline M. temporalis & 59,1 & - & 24 & 54 \\
\hline M. masseter & 54,92 & - & 10 & 54 \\
\hline M. pterygoideus medialis & 30,27 & - & -4 & 30 \\
\hline Force pointed on dentition & 100,02 & - & 20 & -98 \\
\hline
\end{tabular}

Table 2

Standard loads of computer model of $\mathrm{m}$. pterygoideus lateralis (Cartesian coordinate system)

\begin{tabular}{|c|c|c|c|c|c|c|c|c|c|c|}
\hline & & \multicolumn{3}{|c|}{ F (min), $\mathbf{H}$} & \multicolumn{3}{|c|}{$\mathbf{F}(\mathrm{N}), \mathrm{H}$} & \multicolumn{3}{|c|}{ F (max), H } \\
\hline \multirow{6}{*}{$\begin{array}{c}\text { m. pterygoide- } \\
\text { us lateralis }\end{array}$} & \multirow{3}{*}{$\begin{array}{l}\text { Upper belly, } \\
\text { F1 }\end{array}$} & $\mathrm{X}$ & 0 & & $\mathrm{x}$ & 0 & & $\mathrm{x}$ & 0 & \\
\hline & & $\mathrm{Y}$ & -3 & 3,1 & y & -5 & 5,1 & y & -7 & 7,1 \\
\hline & & $\mathrm{Z}$ & 1 & & $\mathrm{z}$ & 1 & & z & 1 & \\
\hline & \multirow{3}{*}{$\begin{array}{l}\text { Lower belly, } \\
\text { F2 }\end{array}$} & $X$ & 0 & & $\mathrm{x}$ & 0 & & $\mathrm{x}$ & 0 & \\
\hline & & Y & -7 & 7,1 & $\mathrm{y}$ & -10 & 10,1 & $\mathrm{y}$ & -13 & 13 \\
\hline & & $\mathrm{Z}$ & -1 & & $\mathrm{z}$ & -1 & & $\mathrm{z}$ & -1 & \\
\hline \multicolumn{2}{|c|}{$\Sigma \mathrm{F}$} & \multicolumn{2}{|c|}{$\Sigma \mathrm{F} 1,2, \mathrm{H}$} & 10,2 & \multicolumn{2}{|c|}{$\Sigma \mathrm{F} 1,2, \mathrm{H}$} & 15,2 & \multicolumn{2}{|c|}{$\Sigma \mathrm{F} 1,2, \mathrm{H}$} & 20,1 \\
\hline
\end{tabular}

In the Ansys software was simulated attachment of several main TMJ ligaments using elastic elements (stiffness was not considered). It was created a grid of discrete volumes (finite element), representing a collection of irregularly shaped objects with complex geometries - 10-node tetrahedral elements with quadratic approximation functions. Conducted calculation of stress-strain state of the mandible and TMJ, rated the distribution of the main stresses and equivalent in Mises stress.

\section{Results of research}

In all cadavers' dissections, we found that lateral pterygoid muscles is fixed to anterior or anteriomedial part of articular disc and capsule (Fig. 2).

During chewing load model was in a complex stress state (tensile strain, compression, bending, and shear). The distribution of stress has been uneven, stress concentration was in sectors of expressed and dense cortical layer: the front of mandible (8,4-11,2 MPa), the posterior edge of branch jaw (9,1-10 MPa), external oblique line (7,3-8,9 MPa), torus mandible and anterior-medial surface of the condyle $(11,4-18,3 \mathrm{MPa})$. More less power of tension arose in the 
chin area (4,8-5,4 MPa), internal surface of mandible (l. mylohyoidea) and point of fixation of m. digastricus (7,1-8,1 MPa) (Fig. 3).

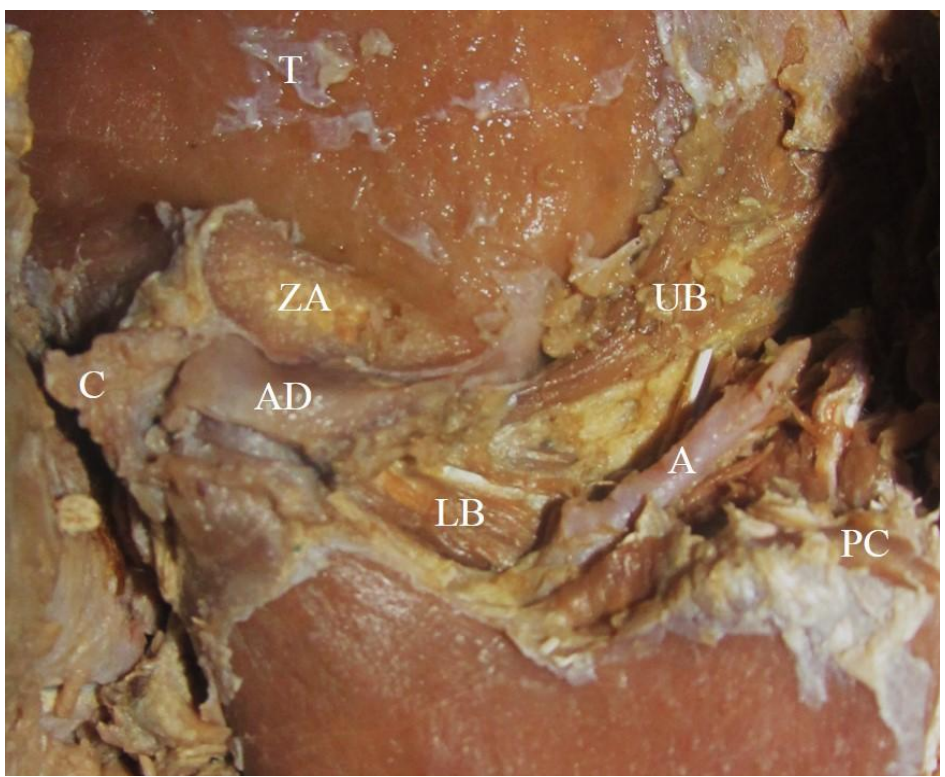

Fig. 2. A lateral view of the lateral section of maxilla-facial area, TMJ structure and LPM (AD - articular disc; C - capsule of TMJ; ZA - zygomatic arch (dissected); UB - upper fascicle of lateral pterygoid muscle; LB - lower fascicle of lateral pterygoid muscle; $\mathrm{PC}$ - processes coronoids (dissected); T - temporal bone; A - a. maxillaris (a. carotis externa))

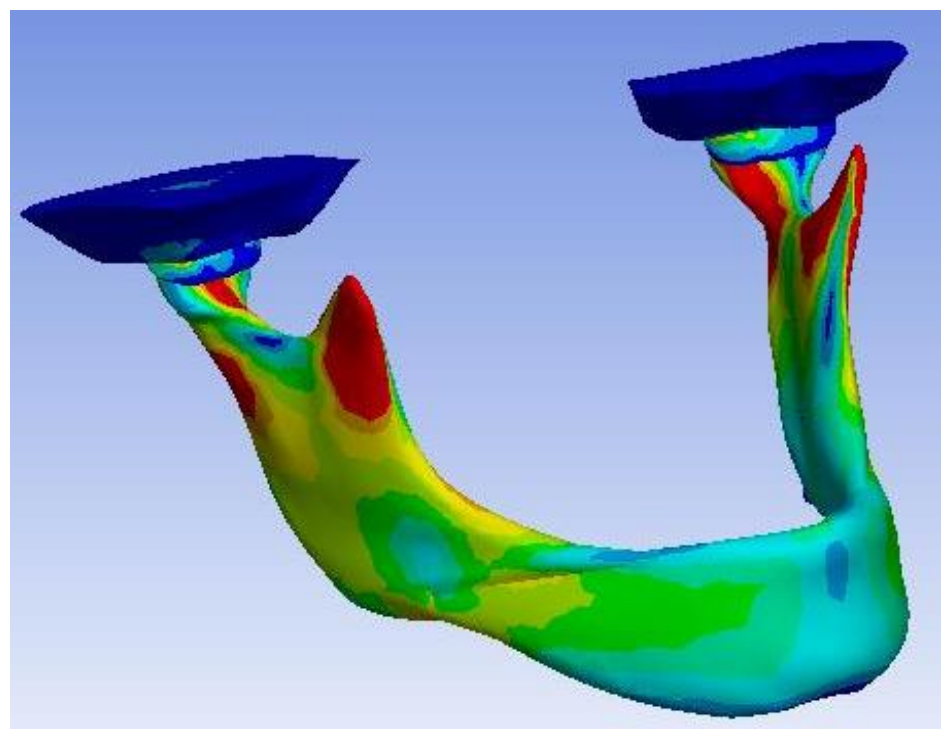

Fig. 3. Distribution Mises equivalent stresses in the mandible

The uneven distribution of stresses in the bone is obvious, because it is well known that the lower jaw is heterogeneous bone structure [19]. These features are contributed to individual physical and mechanical properties and functional features that are inherent in the lower jaw.

The maximum stress was in the front and the distal part of articular disc in the range 1,4-3 MPa and 0,4-1 MPa respectively depend on different conditions of muscular activity. The values of the stress in the lateral, medial and middle parts of the articular disc was from 0,1 to 1,6 $\mathrm{MPa}$, and grew with the increased contraction of lateral pterygoid muscle (Table 3, Fig. 4). 
Table 3

Stress distribution of articular disc depending on contraction force of lateral pterygoid muscle

Force value of LPM, Stress distribution of articular disc of TМJ, МРa*

F, $\mathbf{H}$

Anterior part

Posterior part

\begin{tabular}{ccc}
\hline $\mathrm{F}=0$ & 1,4 & 0,4 \\
$\mathrm{~F}=10(\min )$ & 2 & 0,7 \\
$\mathrm{~F}=15(\mathrm{~N})$ & 2,7 & 0,9 \\
$\mathrm{~F}=20(\max )$ & 3 & 1
\end{tabular}

Note: $*$ - numerical values of maximum stress concentrates

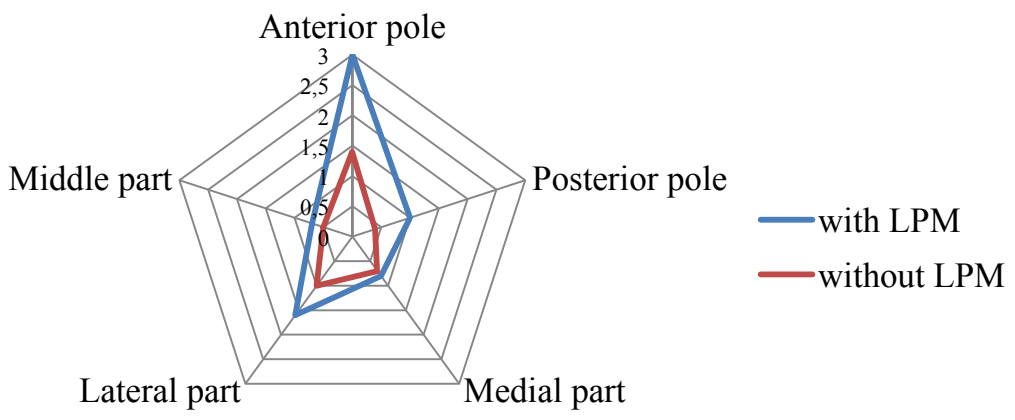

Fig. 4. Comparison of stresses in different parts of the articular disc terminated by the lateral pterygoid muscle $(\mathrm{F}=15 \mathrm{H})$ and without it ( $\mathrm{LPM}$ - lateral pterygoid muscle)

\section{Discussion}

Firstly, researchers investigated static loads on animals (Hohl T. H. and Tucek W. H. [20]; Boyd J. P. et al. [21]). However, many scientists can't extrapolate these data on human organism and widely used mathematical models (Chen J. et al. [22]). Perhaps, this is the next step to develop such new approach, modern occupation in Ukraine as bioengineering [20].

Coming to the evidence, we conducted the division of Mises equivalent stress and strain in different situational positions of masticatory system, taking into account the interaction of components of combined model for the static loading conditions, simulating simultaneous reduction of symmetric state chewing muscles. There was a symmetrical distribution of stress fields and their movement from the chin to condyle of the mandible.

Finite element method is not also a relatively cost and energy saving level of computer science, but the alternative direction of temporomandibular joint study. It helps us to determine mechanisms of development of pathological conditions, using the patient's own three-dimensional model of mandible and TMJ (Fig. 5).

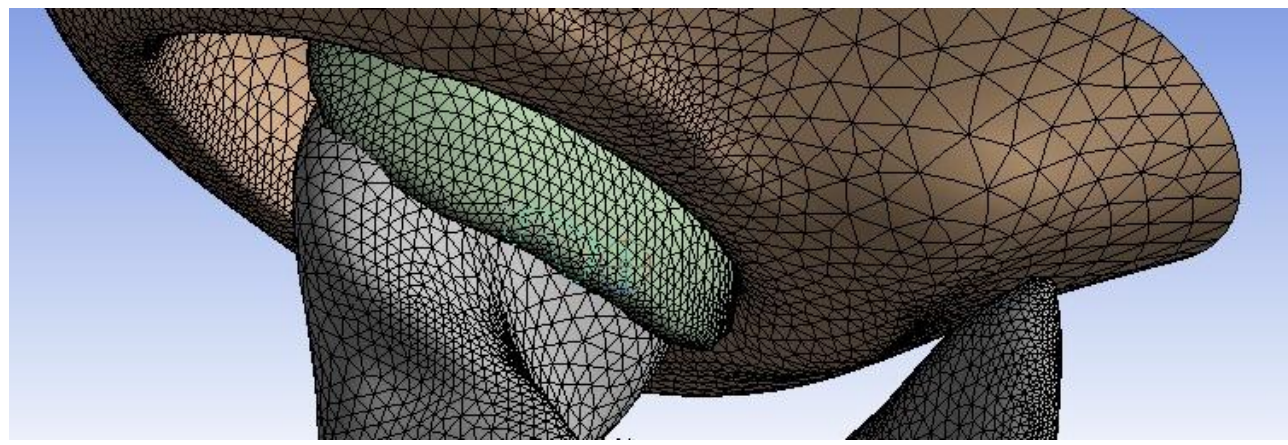

Fig. 5. Finite element model of TMJ 
Total amount of the nodal elements is 1817278 , finite elements -1233096 . It helped us to make necessary calculations and minimized the errors. These results were related with anatomical signs and clinical dysfunction. Well known that contraction of LPM on the one side causes lateral movement, both muscles - an anterior projection or protrusion of the mandible.

According to the review of literature, from $2 \%$ (P. Bravetti et al. [23]) to 69,8 \% (C. J. Tapia et al. [24]) of the upper fascicle of LPM is inserted in articular disc. This fact was confirmed in the result of cadaver's dissection also.

Moreover, the increasing tension in the front of the articular disc is higher than the back part by $41,6 \%$. Enhancing of tension in other parts of the articular disc is equally.

In the beginning of the rotation, and then translational motions of head condylar process the anterior part of articular disc begins to perceive loads. Due to the more dense contact of articular surfaces distally and ligament tension of retrodiscal area, higher loads exist in the distal articular disc.

The appearance of stress concentrations placed in the anterior, posterior and lateral parts of disc coincide with data getting from the direct experiments of articular disc cadaver material. Scientists investigated thinning of layers and perforations of cartilage tissue in some cases (Takamura H., Maruyama T. [25]).

The result of study was submitted on scientific council of the dental faculty of Bogomolets National medical university and author got a master of sciences degree.

\section{Conclusions}

1. Computer model of TMJ accurately reflects the mode of deformation of the joint study of biomechanical systems that allowed determine the stress concentration gradients, the magnitude and direction of the system static conditions deformation.

2. The maximum value of Mises stresses with working chewing muscles corresponds to the topographically buttresses of mandible and are concentrated mainly anteriorly and distally in TMJ disc, equivalent values Mises stresses were 3 and $1 \mathrm{MPa}$ respectively.

3. Taking a treatment of TMJ dysfunction surgeon must investigate the condition of LPM (e. g., using magnetic resonance tomography or electromyography).

\section{References}

[1] Kryshchuk, N. G., Malanchuk, V. A., Kopchak, A. V., Eshchenko, V. O. (2012). Finite-element study of the bone anisotropy and inhomogenity influence on stress distribution in mandible. Naukovi visti NTUU-KPI, 6, 71-76.

[2] Korioth, T. W. P., Versluis, A. (1997). Modeling the Mechanical Behavior of the Jaws and Their Related Structures By Finite Element (Fe) Analysis. Critical Reviews in Oral Biology \& Medicine, 8 (1), 90-104. doi: 10.1177/10454411970080010501

[3] Kalinovsky, D. K., Chuyko, A. N. (2011). Possibilities of the use modern computer technologies CT/CAD/CAM in maxillofacial surgery. Ukr. J. Telemed. Med. Telemat., 9 (1), 35-42.

[4] Leon, L. M. M., Liebgott, B., Agur, A. M., Norwich, K. H. (2006). Computational model of the movement of the human muscles of mastication during opening and closing of the jaw. Computer Methods in Biomechanics and Biomedical Engineering, 9 (6), 387-398. doi: 10.1080/10255840601022734

[5] Citarella, R. (2012). FEM and BEM Analysis of a Human Mandible with Added Temporomandibular Joints. The Open Mechanical Engineering Journal, 6 (1), 100-114. doi: 10.2174/1874155x01206010100

[6] Beek, M., Koolstra, J. H., van Ruijven, L., van Eijden, T. M. G. (2000). Three-dimensional finite element analysis of the human temporomandibular joint disc. Journal of Biomechanics, 33 (3), 307-316. doi: 10.1016/s0021-9290(99)00168-2

[7] Mohammed, S. D., Desai, H. (2014). Basic Concepts of Finite Element Analysis and its Applications in Dentistry: An Overview. Journal of Oral Hygiene \& Health, 02 (05). doi: 10.4172/2332-0702.1000156

[8] Damaris, Ch. (2009). Moving temporomandibular joint research into 21th century. Journal TMJ science, $1,9-18$.

[9] Tanaka, E., Rodrigo, D. P., Tanaka, M., Kawaguchi, A., Shibazaki, T., Tanne, K. (2001). Stress analysis in the TMJ during jaw opening by use of a three-dimensional finite element model based on magnetic resonance images. International Journal of Oral and Maxillofacial Surgery, 30 (5), 421-430. doi: 10.1054/ijom.2001.0132 
[10] Tapia Contreras, J., Cantín, M., Zavando, D., Suazo Galdames, I. (2011). Percentage of Lateral Pterygoid Muscle Inserted in the Disc of Human Temporomandibular Joint. International Journal of Morphology, 29 (3), 965-970. doi: 10.4067/s0717-95022011000300051

[11] Lamela, M. J., Fernandez, P., Dorantes, D., Ramos, A., Tanaka, E., Arguelles, A., Fernandez-Canteli, A. (2012). Experimantal biomechanical characterization and numerical analysis of the temporomandibular joint. Porto, 1-8.

[12] Chen, J., Akyuz, U., Xu, L., Pidaparti, R. M. V. (1998). Stress analysis of the human temporomandibular joint. Medical Engineering \& Physics, 20 (8), 565-572. doi: 10.1016/s1350-4533(98)00070-8

[13] Naeije, M., Hofman, N. (2003). Biomechanics of the Human Temporomandibular Joint during Chewing. Journal of Dental Research, 82 (7), 528-531. doi: 10.1177/154405910308200708

[14] Ciortan, M. (2002). The biomechanical modeling of temporomandibular joint. Ann. of the Oradea Univ., VII, 773-776.

[15] Han, J. J., Hwang, S. J. (2015). Three-dimensional analysis of postoperative returning movement of perioperative condylar displacement after bilateral sagittal split ramus osteotomy for mandibular setback with different fixation methods. Journal of Cranio-Maxillofacial Surgery, 43 (9), 1918-1925. doi: 10.1016/j.jcms.2015.08.004

[16] Tanaka, E., van Eijden, T. (2003). Biomechanical behavior of the temporomandibular joint disc. Critical Reviews in Oral Biology \& Medicine, 14 (2), 138-150. doi: 10.1177/154411130301400207

[17] Shahar, R., Zaslansky, P., Barak, M., Friesem, A. A., Currey, J. D., Weiner, S. (2007). Anisotropic Poisson's ratio and compression modulus of cortical bone determined by speckle interferometry. Journal of Biomechanics, 40 (2), 252-264. doi: 10.1016/j.jbiomech.2006.01.021

[18] Hliňáková, P., Dostálová, T., Daněk, J., Nedoma, J., Hlaváček, I. (2010). Temporomandibular joint and its two-dimensional and three-dimensional modelling. Mathematics and Computers in Simulation, 80 (6), 1256-1268. doi: 10.1016/j.matcom.2009.08.007

[19] Ingawalé, S., Goswami, T. (2009). Temporomandibular Joint: Disorders, Treatments, and Biomechanics. Annals of Biomedical Engineering, 37 (5), 976-996. doi: 10.1007/s10439-009-9659-4

[20] Hohl, T. H., Tucek, W. H. (1982). Measurement of condylar loading forces by instrumented prosthesis in the baboon. Journal of Maxillofacial Surgery, 10, 1-7. doi: 10.1016/s0301-0503(82)80003-9

[21] Brehnan, K., Boyd, R. L., Laskin, J., Gibbs, C. H., Mahan, P. (1981). Direct Measurement of Loads at the Temporomandibular Joint in Macaca arctoides. Journal of Dental Research, 60 (10), 1820-1824. doi: $10.1177 / 00220345810600101501$

[22] Chen, J., Akyuz, U., Xu, L., Pidaparti, R. M. V. (1998). Stress analysis of the human temporomandibular joint. Medical Engineering \& Physics, 20 (8), 565-572. doi: 10.1016/s1350-4533(98)00070-8

[23] El. Haddioui, A., Laison, F., Zouaoui, A., Bravetti, P., Gaudy, J. F. (2005). Functional anatomy of the human lateral pterygoid muscle. Surgical and Radiologic Anatomy, 27 (4), 271-286. doi: 10.1007/ s00276-005-0324-9

[24] Tapia Contreras, J., Cantín, M., Zavando, D., Suazo Galdames, I. (2011). Percentage of Lateral Pterygoid Muscle Inserted in the Disc of Human Temporomandibular Joint. International Journal of Morphology, 29 (3), 965-970. doi: 10.4067/s0717-95022011000300051

[25] Takamura, H., Maruyama, T. (1984). Studies on the structural changes of the temporomandibular joint-macroscopic observations. Nihon Hotetsu Shika Gakkai Zasshi, 28 (1), 49-59. doi: 10.2186/jjps.28.49 